\title{
SOME DOUBLE INTEGRALS INVOLVING MULTIVARIABLE $I$-FUNCTION
}

\author{
D. Kumar, F.Y. Ayant
}

ABstract. A remarkably large number of integral formulas involving diverse special functions have been presented. In this sequel, we aim to establish two double definite integral formulas whose integrands include the multivariable $I$-function. The integral formulas presented here, being very general, are found to reduce to yield a large number of relatively simple integral formulas whose integrands contain various special functions deducible from the multivariable $I$-function, just two of which are demonstrated.

2010 Mathematics Subject Classification: Primary: 33C60, 33C99; Secondary: $44 \mathrm{~A} 20$

Keywords: gamma function, beta function, Legendre polynomial, hypergeometric function, multivariable $I$-function, multivariable $H$-function, double definite integral formulas.

\section{Introduction AND Preliminaries}

Prasad [11] introduced the multivariable $I$-function defined in terms of multiple Mellin-Barnes type integrals

$$
\begin{gathered}
I\left(z_{1}, z_{2}, \ldots, z_{r}\right)=I_{p_{2}, q_{2}, p_{3}, q_{3} ; \cdots ; p_{r}, q_{r}: p^{(1)}, q^{(1)} ; \cdots ; p^{(r)}, q^{(r)}}^{0, n_{2} ; 0, n_{3} ; ; 0, n_{r}: m^{(1)}, n^{(1)} \cdots ; m^{(r)}, n^{(r)}} \\
{\left[\begin{array}{c|cc}
z_{1} & \left(a_{2 j} ; \alpha_{2 j}^{\prime}, \alpha_{2 j}^{\prime \prime}\right)_{1, p_{2}} ; \cdots ; & \left(a_{r j} ; \alpha_{r j}^{(1)}, \cdots, \alpha_{r j}^{(r)}\right)_{1, p_{r}}:\left(a_{j}^{(1)}, \alpha_{j}^{(1)}\right)_{1, p^{(1)}} ; \cdots ;\left(a_{j}^{(r)}, \alpha_{j}^{(r)}\right)_{1, p}(r) \\
\vdots & \left(b_{2 j} ; \beta_{2 j}^{\prime}, \beta_{2 j}^{\prime \prime}\right)_{1, q_{2}} ; \cdots ; & \left(b_{r j} ; \beta_{r j}^{(1)}, \cdots, \beta_{r j}^{(r)}\right)_{1, q_{r}}:\left(b_{j}^{(1)}, \beta_{j}^{(1)}\right)_{1, q^{(1)}} ; \cdots ;\left(b_{j}^{(r)}, \beta_{j}^{(r)}\right)_{1, q^{(r)}}
\end{array}\right]} \\
=\frac{1}{(2 \pi \omega)^{r}} \int_{\mathcal{L}_{1}} \cdots \int_{\mathcal{L}_{r}} \phi\left(s_{1}, \cdots, s_{r}\right) \prod_{i=1}^{r} \phi_{i}\left(s_{i}\right) z_{i}^{s_{i}} \mathrm{~d} s_{1} \cdots \mathrm{d} s_{r} \quad(r \in \mathbb{N}, \omega=\sqrt{-1}),
\end{gathered}
$$


D. Kumar, F.Y. Ayant - Some double integrals involving multivariable...

where

$$
\phi_{i}\left(s_{i}\right)=\frac{\prod_{j=1}^{m^{(i)}} \Gamma\left(b_{j}^{(i)}-\beta_{j}^{(i)} s_{i}\right) \prod_{j=1}^{n^{(i)}} \Gamma\left(1-a_{j}^{(i)}+\alpha_{j}^{(i)} s_{i}\right)}{\prod_{j=m^{(i)}+1}^{q^{(i)}} \Gamma\left(1-b_{j}^{(i)}+\beta_{j}^{(i)} s_{i}\right) \prod_{j=n^{(i)}+1}^{p^{(i)}} \Gamma\left(a_{j}^{(i)}-\alpha_{j}^{(i)} s_{i}\right)}(i=1, \ldots, r)
$$

and

$$
\begin{aligned}
\phi( & \left.s_{1}, \cdots, s_{r}\right) \\
= & \frac{\prod_{j=1}^{n_{2}} \Gamma\left(1-a_{2 j}+\sum_{i=1}^{2} \alpha_{2 j}^{(i)} s_{i}\right) \prod_{j=1}^{n_{3}} \Gamma\left(1-a_{3 j}+\sum_{i=1}^{3} \alpha_{3 j}^{(i)} s_{i}\right) \cdots}{\prod_{j=n_{2}+1}^{p_{2}} \Gamma\left(a_{2 j}-\sum_{i=1}^{2} \alpha_{2 j}^{(i)} s_{i}\right) \prod_{j=n_{3}+1}^{p_{3}} \Gamma\left(a_{3 j}-\sum_{i=1}^{3} \alpha_{3 j}^{(i)} s_{i}\right) \cdots} \\
& \frac{\cdots \prod_{j=1}^{n_{r}} \Gamma\left(1-a_{r j}+\sum_{i=1}^{r} \alpha_{r j}^{(i)} s_{i}\right)}{\cdots \prod_{j=n_{r}+1}^{p_{r}} \Gamma\left(a_{r j}-\sum_{i=1}^{r} \alpha_{r j}^{(i)} s_{i}\right) \prod_{j=1}^{q_{2}} \Gamma\left(1-b_{2 j}-\sum_{i=1}^{2} \beta_{2 j}^{(i)} s_{i}\right)} \\
& \times \frac{1}{\prod_{j=1}^{q_{3}} \Gamma\left(1-b_{3 j}+\sum_{i=1}^{3} \beta_{3 j}^{(i)} s_{i}\right) \cdots \prod_{j=1}^{q_{r}} \Gamma\left(1-b_{r j}-\sum_{i=1}^{r} \beta_{r j}^{(i)} s_{i}\right)} .
\end{aligned}
$$

Here $\Gamma$ is the familiar gamma function (see, e.g., [17, Section 1.1] and, in the following, let $\mathbb{N}$ and $\mathbb{R}^{+}$be the sets of positive integers and positive real numbers, respectively. For the existence and convergence conditions of the multivarible $I$ function (1), we refer to [11]. For example, the condition for absolute convergence of multiple Mellin-Barnes type contour integral (1) can be obtained by extension of the corresponding conditions for multivariable $H$-function as follows:

$$
\left|\arg z_{i}\right|<\frac{1}{2} \Omega_{i} \pi
$$

where, for $i=1, \ldots, r$,

$$
\begin{aligned}
\Omega_{i}= & \sum_{k=1}^{n^{(i)}} \alpha_{k}^{(i)}-\sum_{k=n^{(i)}+1}^{p^{(i)}} \alpha_{k}^{(i)}+\sum_{k=1}^{m^{(i)}} \beta_{k}^{(i)}-\sum_{k=m^{(i)}+1}^{q^{(i)}} \beta_{k}^{(i)} \\
& +\left(\sum_{k=1}^{n_{2}} \alpha_{2 k}^{(i)}-\sum_{k=n_{2}+1}^{p_{2}} \alpha_{2 k}^{(i)}\right)+\cdots+\left(\sum_{k=1}^{n_{r}} \alpha_{r k}^{(i)}-\sum_{k=n_{r}+1}^{p_{r}} \alpha_{r k}^{(i)}\right) \\
& -\left(\sum_{k=1}^{q_{2}} \beta_{2 k}^{(i)}+\sum_{k=1}^{q_{3}} \beta_{3 k}^{(i)}+\cdots+\sum_{k=1}^{q_{r}} \beta_{r k}^{(i)}\right) .
\end{aligned}
$$

We note that the multivariable $I$-function (1) generalizes the multivariable $H$ function (see $[19,20]$ ), which has been investigated by many authors (see, e.g., $[2,3,4,9])$. 
D. Kumar, F.Y. Ayant - Some double integrals involving multivariable...

In this paper, we aim to establish two double integral formulas involving the multivariable $I$-function (1). We also consider some special cases of the two integral formulas.

For simplicity, throughout this paper, we use the following notations:

$$
\begin{aligned}
U & :=p_{2}, q_{2} ; p_{3}, q_{3} ; \cdots ; p_{r-1}, q_{r-1} . \\
V & :=0, n_{2} ; 0, n_{3} ; \cdots ; 0, n_{r-1} . \\
X & :=m^{(1)}, n^{(1)} ; \cdots ; m^{(r)}, n^{(r)} . \\
Y & :=p^{(1)}, q^{(1)} ; \cdots ; p^{(r)}, q^{(r)} . \\
\mathbb{A} & :=\left(a_{2 k} ; \alpha_{2 k}^{(1)}, \alpha_{2 k}^{(2)}\right)_{1, p_{2}} ; \cdots ;\left(a_{(r-1) k} ; \alpha_{(r-1) k}^{(1)}, \alpha_{(r-1) k}^{(2)}, \cdots, \alpha_{(r-1) k}^{(r-1)}\right)_{1, p_{r-1}} . \\
A & :=\left(a_{r k} ; \alpha_{r k}^{(1)}, \alpha_{r k}^{(2)}, \cdots, \alpha_{r k}^{(r)}\right)_{1, p_{r}} . \\
\mathbf{A} & :=\left(a_{k}^{(1)}, \alpha_{k}^{(1)}\right)_{1, p^{(1)}} ; \cdots ;\left(a_{k}^{(r)}, \alpha_{k}^{(r)}\right)_{1, p^{(r)}} \cdot \\
\mathbb{B} & :=\left(b_{2 k} ; \beta_{2 k}^{(1)}, \beta_{2 k}^{(2)}\right)_{1, q_{2}} ; \cdots ;\left(b_{(r-1) k} ; \beta_{(r-1) k}^{(1)}, \beta_{(r-1) k}^{(2)}, \cdots, \beta_{(r-1) k}^{(r-1)}\right)_{1, q_{r-1}} . \\
B & :=\left(b_{r k} ; \beta_{r k}^{(1)}, \beta_{r k}^{(2)}, \cdots, \beta_{r k}^{(r)}\right)_{1, q_{r}} . \\
\mathbf{B} & :=\left(b_{k}^{(1)}, \beta_{k}^{(1)}\right)_{1, q^{(1)}} ; \cdots ;\left(b_{k}^{(r)}, \beta_{k}^{(r)}\right)_{1, q^{(r)}} .
\end{aligned}
$$

\section{REQUiRED INTEGRALS}

Some known integral formulas are recalled in the following lemma.

Lemma 1. Each of the following integral formulas holds.

$$
\begin{aligned}
\int_{0}^{1} x^{\rho} P_{v}(x) \mathrm{d} x & =\frac{\sqrt{\pi} 2^{-\rho-1} \Gamma(\rho+1)}{\Gamma\left(1+\frac{\rho-v}{2}\right) \Gamma\left(\frac{\rho+v+3}{2}\right)} \quad(\Re(\rho)>-1) ; \\
\int_{-1}^{1}(x+1)^{\rho} P_{v}(x) \mathrm{d} x & =\frac{2^{\rho+1} \Gamma^{2}(\rho+1)}{\Gamma(\rho+v+2) \Gamma(\rho-v+1)} \quad(\Re(\rho)>-1) ; \\
\int_{0}^{1} x^{\mu-1}\left(1-x^{\lambda}\right)^{v-1} \mathrm{~d} x & =\frac{1}{\lambda} B\left(\frac{\mu}{\lambda}, v\right) \quad\left(\min \{\Re(\mu), \Re(\nu)\}>0, \lambda \in \mathbb{R}^{+}\right) ;
\end{aligned}
$$


D. Kumar, F.Y. Ayant - Some double integrals involving multivariable...

$$
\begin{gathered}
\int_{0}^{1} x^{\rho-1}(1-x)^{\rho-1}{ }_{2} F_{1}\left[\begin{array}{c}
a, b ; \\
\frac{1}{2}(a+b+1) ; x
\end{array}\right] \mathrm{d} x \\
=\frac{\pi \Gamma(\rho) 2^{-2 \rho+1} \Gamma\left(\frac{1}{2} a+\frac{1}{2} b+\frac{1}{2}\right) \Gamma\left(\rho-\frac{1}{2} a-\frac{1}{2} b+\frac{1}{2}\right)}{\Gamma\left(\frac{1}{2} a+\frac{1}{2}\right) \Gamma\left(\frac{1}{2} b+\frac{1}{2}\right) \Gamma\left(\rho-\frac{1}{2} a+\frac{1}{2}\right) \Gamma\left(\rho-\frac{1}{2} b+\frac{1}{2}\right)} \\
\quad\left(\Re(\rho)>0, \Re\left(\rho+\frac{1-a-b}{2}\right)>0\right),
\end{gathered}
$$

where $P_{v}(x)$ is the Legendre polynomial, $B(\alpha, \beta)$ is the beta function, and ${ }_{2} F_{1}$ is the hypergeometric function.

Proof. For (17), (18), (19), and (20), we refer, for example, respectively, to [7, p. 1187, Entry 7.126-1], [7, p. 1187, Entry 7.127-1], [7, p. 500, Entry 3.251-1], and [10, p. 73 , Eq. (3.1.22)].

\section{MAIn Integrals}

We present two double definite integrals involving the multivariable $I$-function (1) asserted by Theorems 2 and 3 .

Theorem 2. Let $h_{i}, k_{i} \in \mathbb{R}^{+}(i=1, \ldots, r), \Re(\rho)>0, \Re\left(\rho+\frac{1-a-b}{2}\right)>0$, and $\Re(\sigma)>-1$. Also let

$$
\Re(1+\sigma)+\sum_{i=1}^{r} h_{i} \min _{1 \leq j \leq m^{(i)}} \Re\left(\frac{b_{j}^{(i)}}{\beta_{j}^{(i)}}\right)>0, \Re(\rho)+\sum_{i=1}^{r} k_{i} \min _{1 \leq j \leq m^{(i)}} \Re\left(\frac{b_{j}^{(i)}}{\beta_{j}^{(i)}}\right)>0,
$$

and

$$
\Re\left(\frac{1-a-b}{2}+\rho\right)+\sum_{i=1}^{r} k_{i} \min _{1 \leq j \leq m^{(i)}} \Re\left(\frac{b_{j}^{(i)}}{\beta_{j}^{(i)}}\right)>0 .
$$

Further let $\left|\arg \left(z_{i} x^{h_{i}} y^{k_{i}}(1-y)^{k_{i}}\right)\right|<\frac{1}{2} \Omega_{i} \pi(i=1, \ldots, r)$ with $\Omega_{i}$ the same as in (6). Then

$$
\begin{aligned}
& \int_{0}^{1} \int_{0}^{1} x^{\sigma} y^{\rho-1}(1-y)^{\rho-1} P_{v}(x){ }_{2} F_{1}\left[\begin{array}{c}
a, b ; \\
\frac{1}{2}(a+b+1) ; x
\end{array}\right] I\left(\begin{array}{c}
z_{1} x^{h_{1}} y^{k_{1}}(1-y)^{k_{1}} \\
\vdots \\
z_{r} x^{h_{r}} y^{k_{r}}(1-y)^{k_{r}}
\end{array}\right) \mathrm{d} x \mathrm{~d} y \\
& =\frac{\sqrt{\pi^{3}} \Gamma\left(\frac{a+b+1}{2}\right)}{2^{2 \rho+\sigma-1} \Gamma\left(\frac{a+1}{2}\right) \Gamma\left(\frac{b+1}{2}\right)} I_{U: p_{r}+3, q_{r}+4 ; Y}^{V ; 0, n_{r}+3 X}\left(\begin{array}{c|c}
z_{1} 2^{-2 k_{1}-h_{1}} & \mathbb{A}: A_{1}, A_{2}, A_{3}, A: \mathbf{A} \\
\vdots & \vdots \\
z_{r} 2^{-2 k_{r}-h_{r}} & \mathbb{B}: B, B_{1}, B_{2}, B_{3}, B_{4}: \mathbf{B}
\end{array}\right),
\end{aligned}
$$


D. Kumar, F.Y. Ayant - Some double integrals involving multivariable...

where $A_{1}=:\left(-\sigma ; h_{1}, \ldots, h_{r}\right), A_{2}=:\left(1-\rho ; k_{1}, \ldots, k_{r}\right), A_{3}=:\left(\frac{1+a+b}{2}-\rho ; k_{1}, \ldots, k_{r}\right)$, $B_{1}=:\left(\frac{v-\sigma}{2} ; \frac{h_{1}}{2}, \ldots, \frac{h_{r}}{2}\right), B_{2}=:\left(-\frac{v+\sigma+1}{2} ; \frac{h_{1}}{2}, \ldots, \frac{h_{r}}{2}\right)$,

$B_{3}=:\left(-\rho+\frac{1+a}{2} ; k_{1}, \ldots, k_{r}\right)$, and $B_{4}=:\left(-\rho+\frac{1+b}{2} ; k_{1}, \ldots, k_{r}\right)$.

Proof. We denote the left side of $(21)$ by $\mathcal{I}_{1}$. Then expressing the $I$-function of several variables in terms of Mellin-Barnes contour integral with the help of (1), and changing the order of integrations, which is permissible under the stated conditions, we obtain

$$
\begin{aligned}
& \mathcal{I}_{1}=\frac{1}{(2 \pi \omega)^{r}} \int_{\mathcal{L}_{1}} \cdots \int_{\mathcal{L}_{r}} \phi\left(s_{1}, \cdots, s_{r}\right) \prod_{k=1}^{r} \phi_{k}\left(s_{k}\right) z_{k}^{s_{k}}\left[\int_{0}^{1} x^{\sigma+\sum_{i=1}^{r} h_{i} s_{i}} P_{v}(x) \mathrm{d} x\right] \\
& \times\left[\int_{0}^{1} y^{\rho-1+\sum_{i=1}^{r} k_{i} s_{i}}(1-y)^{\rho-1+\sum_{i=1}^{r} k_{i} s_{i}}{ }_{2} F_{1}\left[\begin{array}{r}
a, b ; \\
\frac{1}{2}(a+b+1) ;
\end{array}\right] \mathrm{d} y\right] \mathrm{d} s_{1} \cdots \mathrm{d} s_{r} .
\end{aligned}
$$

We evaluate the inner $x$ - and $y$-integrals in (22) with the help of the integral formulas in Lemma 1 and obtain

$$
\int_{0}^{1} x^{\sigma+\sum_{i=1}^{r} h_{i} s_{i}} P_{v}(x) \mathrm{d} x=\frac{\sqrt{\pi} 2^{-\sigma-1-\sum_{i=1}^{r} h_{i} s_{i}} \Gamma\left(\sigma+1+\sum_{i=1}^{r} h_{i} s_{i}\right)}{\Gamma\left(1+\frac{\sigma-v}{2}+\frac{\sum_{i=1}^{r} h_{i} s_{i}}{2}\right) \Gamma\left(\frac{\sigma+v+3}{2}+\frac{\sum_{i=1}^{r} h_{i} s_{i}}{2}\right)}
$$

and

$$
\begin{aligned}
& \int_{0}^{1} y^{\rho-1+\sum_{i=1}^{r} k_{i} s_{i}}(1-y)^{\rho-1+\sum_{i=1}^{r} k_{i} s_{i}}{ }_{2} F_{1}\left[\begin{array}{r}
a, b ; \\
\frac{1}{2}(a+b+1) ;
\end{array}\right] \mathrm{d} y \\
& =\frac{\pi \Gamma\left(\frac{a+b+1}{2}\right)}{2^{2 \rho+\sigma-1} \Gamma\left(\frac{a+1}{2}\right) \Gamma\left(\frac{b+1}{2}\right)} \frac{\Gamma\left(\rho+\sum_{i=1}^{r} k_{i} s_{i}\right) \Gamma\left(\rho-\frac{1}{2} a-\frac{1}{2} b+\frac{1}{2}+\sum_{i=1}^{r} k_{i} s_{i}\right)}{2^{2 \sum_{i=1}^{r} k_{i} s_{i}} \Gamma\left(\rho-\frac{1}{2} a+\frac{1}{2}+\sum_{i=1}^{r} k_{i} s_{i}\right) \Gamma\left(\rho-\frac{1}{2} b+\frac{1}{2}+\sum_{i=1}^{r} k_{i} s_{i}\right)} .
\end{aligned}
$$

Finally, substituting the equations (23) and (24) in (22) and reinterpreting the resulting multiple Mellin-Barnes contour integral in terms of $I$-function of $r$-variables, we obtain the desired result (21).

Theorem 3. Let $h_{i}, k_{i} \in \mathbb{R}^{+}(i=1, \ldots, r), \lambda \in \mathbb{R}^{+}, \Re(v)>0, \Re(\rho)>0$, and $\Re(\sigma)>-1$. Also let

$$
\Re(1+\sigma)+\sum_{i=1}^{r} h_{i} \min _{1 \leq j \leq m^{(i)}} \operatorname{Re}\left(\frac{b_{j}^{(i)}}{\beta_{j}^{(i)}}\right)>0, \Re(\tau)+\sum_{i=1}^{r} k_{i} \min _{1 \leq j \leq m^{(i)}} \operatorname{Re}\left(\frac{b_{j}^{(i)}}{\beta_{j}^{(i)}}\right)>0,
$$


D. Kumar, F.Y. Ayant - Some double integrals involving multivariable...

and

$$
\Re\left(\frac{\tau}{\lambda}\right)+\sum_{i=1}^{r} \frac{k_{i}}{\lambda} \min _{1 \leq j \leq m^{(i)}} \Re\left(\frac{b_{j}^{(i)}}{\beta_{j}^{(i)}}\right)>0 .
$$

Further let

$$
\left|\arg \left[z_{i}(1-x)^{h_{i}} y^{k_{i}}\left(1-y^{\lambda}\right)^{k_{i}}\right]\right|<\frac{1}{2} \Omega_{i} \pi,
$$

where $\Omega_{i}(i=1, \ldots, r)$ are the same in $(6)$. Then

$$
\begin{gathered}
\int_{-1}^{1} \int_{0}^{1}(x+1)^{\sigma} y^{\tau-1}\left(1-y^{\lambda}\right)^{\tau-1} P_{v}(x) I\left(\begin{array}{c}
z_{1}(1-x)^{h_{1}} y^{k_{1}}\left(1-y^{\lambda}\right)^{k_{1}} \\
\vdots \\
z_{r}(1-x)^{h_{r}} y^{k_{r}}\left(1-y^{\lambda}\right)^{k_{r}}
\end{array}\right) \mathrm{d} x \mathrm{~d} y \\
=\frac{2^{\sigma+1}}{\lambda} I_{U: p_{r}+4, q_{r}+3 ; Y}^{V ; 0, n_{r}+4 ; X}\left(\begin{array}{c|c}
z_{1} 2^{h_{1}} & \mathbb{A}: C_{1}, C_{1}, C_{2}, C_{3}, A: \mathbf{A} \\
\vdots & \vdots \\
z_{r} 2^{h_{r}} & \mathbb{B}: B, D_{1}, D_{2}, D_{3}: \mathbf{B}
\end{array}\right)
\end{gathered}
$$

where $C_{1}:=\left(-\sigma ; h_{1}, \ldots, h_{r}\right), C_{2}=:\left(1-\frac{\tau}{\lambda} ; \frac{k_{1}}{\lambda}, \ldots, \frac{k_{r}}{\lambda}\right), C_{3}=:\left(1-\tau ; k_{1}, \ldots, k_{r}\right)$, $D_{1}:=\left(-1-\sigma-v ; h_{1}, \ldots, h_{r}\right), D_{2}=:\left(v-\sigma ; h_{1}, \ldots, h_{r}\right), D_{3}=:\left(1-\tau-\frac{\tau}{\lambda} ; \frac{k_{1}}{\lambda}, \ldots, \frac{k_{r}}{\lambda}\right)$.

Proof. A similar argument as in the proof of Theorem 3 will establish the result here. We omit the details.

\section{Special CASES AND REMARKS}

The integral formulas in Theorems 2 and 3, being very general, can be specialized to yield a number of relatively simple integral formulas involving certain special functions reducible from the multivariable $I$-function (1). Here we demonstrate only two integral formulas in the following corollaries.

To do this, let $U=V=\mathbb{A}=\mathbb{B}=0$. Then the multivariable $I$-function reduces to the multivariable $H$-function (see, e.g., $[2,3,4,9,11]$ ). Also let

$$
\begin{gathered}
\Omega_{i}^{\prime}=: \sum_{k=1}^{n^{(i)}} \alpha_{k}^{(i)}-\sum_{k=n^{(i)}+1}^{p^{(i)}} \alpha_{k}^{(i)}+\sum_{k=1}^{m^{(i)}} \beta_{k}^{(i)}-\sum_{k=m^{(i)}+1}^{q^{(i)}} \beta_{k}^{(i)}+\sum_{k=1}^{n_{s}} \alpha_{s k}^{(i)}-\sum_{k=n_{s}+1}^{p_{s}} \alpha_{s k}^{(i)}-\sum_{k=1}^{q_{s}} \beta_{s k}^{(i)} \\
(i=1, \ldots, r) .
\end{gathered}
$$

We consider only two special cases of the main results, given in Corollaries 4 and 5 . 
D. Kumar, F.Y. Ayant - Some double integrals involving multivariable...

Corollary 4. Let $\left|\arg \left(z_{i} x^{h_{i}} y^{k_{i}}(1-y)^{k_{i}}\right)\right|<\frac{1}{2} \Omega_{i}^{\prime} \pi(i=1, \ldots, r)$ with $\Omega_{i}^{\prime}$ the same as in (26). Also let the other conditions and notations be the same as in Theorem 2. Then

$$
\begin{gathered}
\int_{-1}^{1} \int_{0}^{1}(x+1)^{\sigma} y^{\tau-1}\left(1-y^{\lambda}\right)^{\tau-1} P_{v}(x){ }_{2} F_{1}\left[\begin{array}{c}
a, b ; \\
\frac{1}{2}(a+b+1) ; x
\end{array}\right] H\left[\begin{array}{c}
z_{1} x^{h_{1}} y^{k_{1}}(1-y)^{k_{1}} \\
\vdots \\
z_{r} x^{h_{r}} y^{k_{r}}(1-y)^{k_{r}}
\end{array}\right] \mathrm{d} x \mathrm{~d} y \\
=\frac{\sqrt{\pi^{3}} \Gamma\left(\frac{a+b+1}{2}\right)}{2^{2 \rho+\sigma-1} \Gamma\left(\frac{a+1}{2}\right) \Gamma\left(\frac{b+1}{2}\right)} H_{p_{r}+3, q_{r}+4 ; Y}^{0, n_{r}+3 ; X}\left[\begin{array}{c|c}
z_{1} 2^{-2 k_{1}-h_{1}} & A_{1}, A_{2}, A_{3}, A: \mathbf{A} \\
\vdots & \vdots \\
z_{r} 2^{-2 k_{r}-h_{r}} & B, B_{1}, B_{2}, B_{3}, B_{4}: \mathbf{B}
\end{array}\right] .
\end{gathered}
$$

Corollary 5. Let

$$
\left|\arg \left[z_{i}(1-x)^{h_{i}} y^{k_{i}}\left(1-y^{\lambda}\right)^{k_{i}}\right]\right|<\frac{1}{2} \Omega_{i}^{\prime} \pi
$$

where $\Omega_{i}^{\prime}(i=1, \ldots, r)$ are the same in (26). Also let the other conditions and notations be the same as in Theorem 3. Then

$$
\begin{gathered}
\int_{-1}^{1} \int_{0}^{1}(x+1)^{\sigma} y^{\tau-1}\left(1-y^{\lambda}\right)^{\tau-1} P_{v}(x) H\left[\begin{array}{c}
z_{1}(1-x)^{h_{1}} y^{k_{1}}\left(1-y^{\lambda}\right)^{k_{1}} \\
\vdots \\
z_{r}(1-x)^{h_{r}} y^{k_{r}}\left(1-y^{\lambda}\right)^{k_{r}}
\end{array}\right] \mathrm{d} x \mathrm{~d} y \\
=\frac{2^{\sigma+1}}{\lambda} H_{p_{r}+4, q_{r}+3 ; Y}^{0, n_{r}+4 ; X}\left[\begin{array}{c|c}
z_{1} 2^{h_{1}} & C_{1}, C_{1}, C_{2}, C_{3}, A: \mathbf{A} \\
\vdots & \vdots \\
z_{r} 2^{h_{r}} & B, D_{1}, D_{2}, D_{3}: \mathbf{B}
\end{array}\right] \cdot \quad \text { (28) }
\end{gathered}
$$

A similar argument given above will establish a large number of integral formulas whose integrand include diverse special functions such as the multivariable $A$-function (see [6]), the multivariable Aleph-function (see [1]), the Aleph-function of two variables (see [8]; see also [15]), the $I$-function of two variables (see [16]), the $H$-function of two variables (see [18]; see also [13]), the Aleph-function of one variable (see $[21,22]$ ), the $I$-function of one variable (see [14]), the $A$-function of one variable (see [5]).

\section{Conclusion}

We established two double definite integral formulas whose integrands include the multivariable $I$-function. The integral formulas presented here, being very general, are found to reduce in a large number of relatively simple integral formulas 
D. Kumar, F.Y. Ayant - Some double integrals involving multivariable...

whose integrands contain various special functions deducible from the multivariable $I$-function. The multivariable $I$-function can also be suitably specialized to yield a large number of special functions of one or several variables or product of several such special functions which are expressible in terms of $E, G$ and $H$-functions of one, two and more variables.

\section{REFERENCES}

[1] F.Y. Ayant, An integral associated with the Aleph-functions of several variables, Int. J. Math. Trends Tech. 31, 3 (2016), 142-154.

[2] D. Baleanu, D. Kumar and S. D. Purohit, Generalized fractional integrals of product of two $H$-functions and a general class of polynomials, Int. J. Comput. Math. 93, 8 (2016), 1320-1329.

[3] J. Choi, J. Daiya, D. Kumar and R. K. Saxena, Fractional differentiation of the product of Appell function $F_{3}$ and multivariable $H$-function, Commun. Korean Math. Soc. 31, 1 (2016), 115-129.

[4] J. Daiya, J. Ram and D. Kumar, The multivariable $H$-function and the general class of Srivastava polynomials involving the generalized Mellin-Barnes contour integrals, FILOMAT 30, 6 (2016), 1457-1464.

[5] B. P. Gautam and A. S. Asga, The A-Function, Revista Mathematica, Tucuman, 1980.

[6] B. P. Gautam, A. S. Asgar and A. N. Goyal, On the multivariable A-function, Vijnana Parishas Anusandhan Patrika 29, 4 (1986), 67-81.

[7] I.S. Gradshteyn and I.M. Ryzhik, Table of Integrals, Series, and Products, Academic Press, New York, 1980.

[8] D. Kumar, Generalized fractional differintegral operators of the Aleph-function of two variables, J. Chemical, Biol. Phys. Sci.- Section C 6, 3 (2016), 1116-1131.

[9] D. Kumar, S. D. Purohit and J. Choi, Generalized fractional integrals involving product of multivariable $H$-function and a general class of polynomials, J. Nonlinear Sci. Appl. 9 (2016), 8-21.

[10] A. M. Mathai and R. K. Saxena, Generalized Hypergeometric Functions with Applications in Statistics and Physical Sciences, Springer-Verlag, Berlin, Heidelberg and New York, 1973.

[11] Y. N. Prasad, Multivariable I-function, Vijnana Parishad Anusandhan Patrika 29 (1986), 231-237.

[12] J. Prathima, V. Nambisan and S. K. Kurumujji, A study of I-function of several complex variables, Internat. J. Engrg. Math. 2014 (2014), 1-12. 
D. Kumar, F.Y. Ayant - Some double integrals involving multivariable...

[13] J. Ram and D. Kumar, Generalized fractional integration involving Appell hypergeometric function of the product of two H-functions, Vijanana Parishad Anusandhan Patrika 54, 3 (2011), 33-43.

[14] V.P. Saxena, Formal solution of certain new pair of dual integral equations involving H-function, Proc. Nat. Acad. Sci. India Sect. A 51 (2001), 366-375.

[15] R.K. Saxena, J. Ram and D. Kumar, Generalized fractional integral of the product of two Aleph-functions, Appl. Appl. Math. 8, 2 (2013), 631-646.

[16] C.K. Sharma and P.L. Mishra, On the I-function of two variables and its properties, Acta Ciencia Indica Math. 17 (1991), 667-672.

[17] H.M. Srivastava and J. Choi, Zeta and q-Zeta Functions and Associated Series and Integrals, Elsevier Science Publishers, Amsterdam, London and New York, 2012.

[18] H.M. Srivastava, K.C. Gupta and S.P. Goyal, The H-function of One and Two variables with Applications, South Asian Publishers, New Delhi and Madras, 1982.

[19] H.M. Srivastava and R. Panda, Some expansion theorems and generating relations for the $H$-function of several complex variables, Comment. Math. Univ. St. Paul. 24 (1975), 119-137.

[20] H.M. Srivastava and R. Panda, Some expansion theorems and generating relations for the $H$-function of several complex variables, Comment. Math. Univ. St. Paul. 24 (1975), 119-137.

[21] N. Südland, B. Baumann and T. F. Nannenmacher, Open problem: Who knows about the -function?, Fract. Calc. Appl. Anal. 1, 4 (1998), 401-402.

[22] N. Südland, B. Baumann and T. F. Nannenmacher, Fractional driftless FokkerPlanck equation with power law diffusion coefficients in V. G. Gangha, E. W. Mayr, W. G. Vorozhtsov (Eds.), Computer Algebra in Scientific Computing (CASC Konstanz 2001), Springer, Berlin, 2001, 513-525.

Dinesh Kumar

Department of Applied Sciences, College of Agriculture, Sumerpur-Pali, Agriculture University of Jodhpur, Jodhpur 342304, India email: dinesh_dino03@yahoo.com

Frédéric Ayant

Collége Jean L'herminier, Allée des Nymphéas, 83500 La Seyne-sur-Mer, France; ${ }^{2}$ Six-Fours-les-Plages 83140, Department of Var, France email: fredericayant@gmail.com 